\title{
Vacuum polarization in the model of Dirac fermions with anoma- lous magnetic moment interacting with background axial-vector condensate and magnetic field
}

\author{
A. F. Bubnov ${ }^{1, \star}$, N. V. Gubina ${ }^{1}$, V. Ch. Zhukovsky ${ }^{1, \star \star}$ \\ ${ }^{1}$ Department of Theoretical Physics, Faculty of Physics, Moscow State University, 119991 Moscow, Russia
}

\begin{abstract}
In this paper, we consider vacuum polarization effects in the model of charged fermions with anomalous magnetic moment and axial-vector interaction term in a uniform magnetic field. Nontrivial orrections to the effective Lagrangian from the anomalous moment and axial-vector term are calculated with account for various configurations of parameters of the model.
\end{abstract}

\section{Introduction}

As is known a charged particle described by the Dirac equation should possess an intrinsic moment, i.e., spin, and also a kinematic Dirac magnetic moment whose value is equal to the Bohr magneton $\mu_{0}=\frac{e \hbar}{2 m c}$. In the lowest order with respect to the fine-structure constant $\alpha=\frac{e^{2}}{\hbar c}$ (Schwinger [1]), the total value of the electron magnetic moment with account fot the vacuum contribution becomes

$$
\mu_{S c h}=\mu_{0}+\Delta \mu_{S c h}, \Delta \mu_{S c h}=\frac{\alpha}{2 \pi} \cdot \mu_{0},
$$

where $\Delta \mu_{S c h}$ is the Schwinger vacuum magnetic moment of the electron, which was shown [2] to depend on the electron energy and the strength of the external magnetic field. In the general case, a Dirac particle can obtain an anomalous magnetic moment (AMM) $\Delta \mu$ not necessarily equal to its vacuum value $\Delta \mu_{S c h}$ ([3]). Then the total magnetic moment of a Dirac particle is equal to

$$
\mu_{\text {tot }}=\mu_{0}+\Delta \mu, \Delta \mu \simeq \kappa \mu_{0} .
$$

Here $\kappa$, a 4-invariant quantity, is the value of the anomalous moment, which is assumed to be the given constant of the model (in the case of the vacuum magnetic moment of the electron $\kappa=\frac{\alpha}{2 \pi}$ ). The interaction of the anomalous magnetic moment with an external electromagnetic field $F_{\alpha \beta}$ can be described phenomenologically by adding the Pauli-Schwinger term $\Delta \mu \frac{1}{2} \sigma^{\alpha \beta} F_{\alpha \beta}$ [4] (see also [5]) to the Dirac Lagrangian. Along with the AMM, another additional parameter can be taken into account in the general description of fermion. This new term characterizes the violation of Lorentz invariance in the system. The Standard Model does not include a mechanism that allows the violation of Lorentz and CPT symmetries. These violations, however, can be present in extended models related to theories

\footnotetext{
$\star$ e-mail: bfandrey@mail.ru

$\star \star$ e-mail: zhukovsk@phys.msu.ru
} 
including terms of interaction with axial-vector condensate discussed in connection with possible manifestations of a fermion induced torsion field [6]. or some more fundamental theories valid in higher dimensions. Thus, for example, such terms can occur in gravitation theory and cosmology (see [7],[8]), where the violation of symmetries is connected with the spatial anisotropy that is caused by some vector field with a nonzero vacuum average, or, as follows from [9], in the string theory, in which it is assumed that our world is situated on a brane that exists in a universe of a larger number of dimensions. The resulting theory can be effectively described in the framework of the Standard Model Extension (SME) [10, 11].

In this paper, we calculate a one-loop effective Lagrangian of electromagnetic field in the framework of the SME, generalized by introducing the Pauli-Schwinger AMM term in the Dirac Lagrangian for a fermion in a constant and uniform magnetic field.

We introduce an additional CPT-odd term to the Dirac equation of the form $\bar{\psi} \gamma^{5} \gamma^{\mu} b_{\mu} \psi$, where $b^{\mu}$ is the constant axial four-vector [12-14]). The Dirac equation of this form and the energy spectrum for a fermion were considered earlier [15]. Modern experiments give the following estimates (see, e.g. [16]): $\left|b_{0}\right| \lesssim 10^{-14} \mathrm{GeV},|\vec{b}| \lesssim 10^{-31} \mathrm{GeV}$.

This calculation of the effective Lagrangian is motivated by the study of the so called chiral magnetic effect [17] with the vacuum current induced by an external magnetic field in the presence of the chiral chemical potential, which is similar to the above mentioned Lorentz-violating term.

One should also note recent studies of interaction of massless fermions with the background field, when the chirality of massless fermions makes it possible to observe an energy current along the external magnetic field, or the so-called axial magnetic effect, which helps to explain the low energy description of Weyl semi-metals conductive properties [18], [19]. The axial magnetic effect in QCD is also examined with respect to phase structure of quark-gluon plasma and the effect of deconfinment [20]. Note that the problem of the description of the dynamics of fermions that possess an anomalous moment has attracted considerable attention recently (see, e.g. [3], [21], [22], [23]. Background interaction of the form of the Pauli-Schwinger term can also occur in the framework of the SME and in parity-violating interactions of cosmic fields with atoms, molecules, and nuclei [3] ${ }^{1}$.

\section{Energy spectrum of electron}

Consider a charged fermion in a constant uniform magnetic field in the presence of an additional background axial-vector interaction of the form $\bar{\psi} \gamma^{5} \gamma^{\mu} b_{\mu} \psi$ with the constant axial vector $b^{\mu}$ in the Lagrangian of the model.

In this paper, we consider two variants: the axial fourvector $b^{\mu}$ has the form $\left(b^{0}, \mathbf{0}\right)$ or $(0, \mathbf{b})$ (only the time or space components are nonzero, an evident generalization with both components nonvanishing is also presented). In the latter case we assume that the three-dimensional vector $\mathbf{b}$ is aligned along (or opposite to) the magnetic-field strength vector $\mathbf{H}$.

In the former case, we also take the condensate to include the fermion AMM $\kappa \mu_{0}$ (2) (which can occur for cosmic fields that violate the interaction parity [3]).

The total Lagrangian of a negatively charged Dirac fermion that interacts with the above condensates has the following form:

$$
\mathcal{L}=\bar{\psi}\left(i \gamma^{\mu} D_{\mu}-m\right) \psi-\bar{\psi} \gamma^{5} \gamma^{\mu} b_{\mu} \psi+\bar{\psi}\left(\frac{\Delta \mu}{2} \sigma^{\alpha \beta} F_{\alpha \beta}\right) \psi,
$$

\footnotetext{
${ }^{1}$ It should also be noted that the influence of CPT-odd action on the dipole moment of bound electrons that results in the appearance of an anomalous moment of atomic orbitals and specific asymmetry of the angular distribution of radiation for a hydrogen-like atom was considered in the framework of the SME [24].
} 
where the first term is the standard Dirac Lagrangian, the second part corresponds to the interaction with the axial-vector background that violates the Lorentz invariance of the theory and, as can be easily verified, CPT parity, and the last term is the part that phenomenologically describes the interaction of the AMM $\Delta \mu$ of the Dirac particle with the electromagnetic field.

The stationary solutions of the equation, following from the total Lagrangian (3)

$$
\left(i \gamma^{\mu} D_{\mu}-m+\frac{\Delta \mu}{2} \sigma^{\mu \nu} F_{\mu \nu}-\gamma^{5} \gamma^{\alpha} b_{\alpha}\right) \psi=0
$$

where $\sigma^{\mu \nu}=\frac{i}{2}\left(\gamma^{\mu} \gamma^{\nu}-\gamma^{v} \gamma^{\mu}\right)$, and $F_{\mu \nu}$ is a constant and uniform external field, will be found in the case, when an external constant uniform magnetic field with the strength $\mathbf{H}$, is directed along the $z$ axis: $\mathbf{H}=H \mathbf{e}_{z}, H>0$, and the electric field is absent. Consider the following possible particular cases (in what follows, $n=0,1,2, \ldots$ is the Landau principal quantum number, $\zeta= \pm 1$ is the fermion spin projection on the magnetic field direction, $\epsilon= \pm$ is the sign of the energy).

a) $\mathbf{A M M} \Delta \mu=0$, axial condensate $b_{0} \neq 0, \vec{b}=0\left([15],[17],[25],[26],[27]^{2}\right)$

$$
E^{2}=m^{2}+\left(\operatorname{sgn}(p) \sqrt{p^{2}+2 e H n}+\zeta b_{0}\right)^{2} .
$$

b) AMM $\Delta \mu=0$, axial condensate $b_{0}=0, \vec{b} \neq 0, \vec{b}=(0,0, b), p \equiv p_{z}([15],[28])$

$$
E^{2}=2 e H n+\left(\sqrt{m^{2}+p^{2}}+\zeta b\right)^{2} .
$$

c) $\mathbf{A M M} \Delta \mu \neq 0$, axial condensate $b_{0}=0, \vec{b}=0$ ([15], [28], [29])

$$
E^{2}=p^{2}+\left(\sqrt{2 e H n+m^{2}}+\zeta \Delta \mu H\right)^{2}
$$

d) $\mathbf{A M M} \Delta \mu \neq 0$, axial condensate, $b_{0} \neq 0, \vec{b}=0([15])$

$$
E^{2}=m^{2}+p^{2}+2 e H n+(\Delta \mu H)^{2}+b_{0}^{2} \pm 2 \sqrt{\left[m(\Delta \mu H)+b_{0} p\right]^{2}+2 e H n\left[(\Delta \mu H)^{2}+b_{0}^{2}\right]} .
$$

\section{The effective Lagrangian}

The effective Lagrangian can be calculated basing upon the proper time (fifth parameter) method [30]

$$
\mathcal{L}_{\text {eff }}=\frac{1}{4 \sqrt{\pi}} \frac{e H}{(2 \pi)^{2}} \int_{-\infty}^{\infty} d p \sum_{n, \zeta, \epsilon} \int_{\frac{1}{\Lambda^{2}}}^{\infty} \frac{d s}{s^{3 / 2}} \mathrm{e}^{-s E^{2}},
$$

where $1 / \Lambda^{2}$ is the cutoff parameter. In this formula summation over the quantum numbers gives

$$
\sum_{n, \zeta, \epsilon} \mathrm{e}^{-2 s(e H) n}=2\left[1+2 \sum_{n=1}^{\infty} \mathrm{e}^{-2 s(e H) n}\right]=2 \operatorname{coth}(e H s) .
$$

\footnotetext{
${ }^{2}$ Formally speaking, in this paper, the axial vector $b_{0}$ is considered in place of the chiral chemical potential $\mu_{5}$, which was used in [17], [25] for description of the quark spectrum in the case of asymmetry with respect to the number of left and right quarks due to axial anomaly.
} 
Here, the first term corresponds to the quantum numbers of the ground (nondegenerate) state $n=0, \zeta=+1$ (for $e>0$ ) and $n=0, \zeta=-1$ (for $e<0$ ). As a result, with $b_{\mu}=0$ and $\Delta \mu=$ 0 , after regularization and renormalization one obtains the well-known Heisenberg-Euler effective Lagrangian (see, e.g., $[1,5]$ )

$$
\mathcal{L}_{\text {eff }}=-\frac{1}{8 \pi^{2}} \int_{0}^{\infty} \frac{d s}{s} \mathrm{e}^{-s m^{2}}\left[(e H)(e E) \cot (e s E) \operatorname{coth}(e s H)-\frac{1}{s^{2}}+\frac{1}{3}\left((e E)^{2}-(e H)^{2}\right)\right] .
$$

In what follows we shall use the above formulated particular solutions for the Dirac energy eigenvalues (5), (6), (7) and (8).

a) Consider the eigenvalue solution $\Delta \mu=0, \vec{b}=0, b_{0} \neq 0$

In the approximation of small parameter $b_{0} \ll 1$ in the expansion in powers of $b_{0}$ the linear term will be absent due to the oddness of the expression in $\zeta$ for $n \neq 0$, and at $n=0$ due to oddness in the integration variable $p$ that in this case enters the energy in combination $(p+\zeta b)^{2}$ (at fixed $\zeta$ ). Then expansion in $b_{0}$ of expression (9) after summation over all quantum numbers (10) gives

$$
\Delta \mathcal{L}^{b}=b_{0}^{2} \frac{(e H)^{2}}{(2 \pi)^{2}} \int_{\frac{1}{\Lambda^{2}}}^{\infty} d s \frac{\mathrm{e}^{-s m^{2}}}{\sinh ^{2}(e H s)}
$$

Integrating by parts and performing regularization we arrive at the expression for the $b_{0}^{2}$ contribution to the effective Lagrangian

$$
\Delta \mathcal{L}^{b}=\frac{b_{0}^{2}}{4 \pi^{2}}\left[-m^{2} e H \int_{0}^{\infty} d s \mathrm{e}^{-s m^{2}}\left(\operatorname{coth}(e H s)-\frac{1}{e H s}\right)+\Lambda^{2}-m^{2} \ln \frac{\Lambda^{2}}{m^{2}}\right] .
$$

Note that the terms with the cut-off $\Lambda$ do not actually depend on the magnetic field, and thus can be eliminated by subtraction, yielding the final result

$$
\Delta \mathcal{L}^{b}=-b_{0}^{2} \frac{(e H) m^{2}}{4 \pi^{2}} \int_{0}^{\infty} d s \mathrm{e}^{-s m^{2}}\left(\operatorname{coth}(e H s)-\frac{1}{e H s}\right) .
$$

In the weak field limit, $e H \ll m^{2}$ after integration one obtains

$$
\Delta \mathcal{L}=\frac{1}{12 \pi^{2}} b_{0}^{2}(e H)^{2} \int_{0}^{\infty} d x \mathrm{e}^{-x} \frac{x}{m^{2}}=\frac{b^{2}(e H)^{2}}{12 \pi^{2} m^{2}} .
$$

In the strong field limit, $e H \gg m^{2}$, using the substitution $x=s m^{2}$ and integrating approximately

$$
\int_{0}^{\infty} d x \mathrm{e}^{-x}\left(\operatorname{coth}\left(e H x / m^{2}\right)-\frac{1}{e H x / m^{2}}\right) \approx 1,
$$

one gets the result

$$
\Delta \mathcal{L} \approx \frac{b_{0}^{2} e H}{4 \pi^{2}} .
$$

A more accurate approximation is obtained if the results are represented via Euler $\psi$-function (see [27]).

$$
\Delta \mathcal{L}=-\frac{b_{0}^{2}}{4 \pi^{2}} m^{2}\left(\psi\left(\frac{m^{2}}{2 H}\right)-\ln \left(\frac{m^{2}}{2 H}\right)+\frac{H}{m^{2}}\right)
$$


b) Consider the eigenvalue solution $\Delta \mu=0, \vec{b}=(0,0, b), b \neq 0, b_{0}=0$

In the approximation of small $b$ the expansion is considered up to $b^{2}$ only (the linear term is absent since the expression is odd with respect to $\zeta$ ).

The final renormalized expression takes the form

$$
\Delta \mathcal{L}^{b}=b^{2} \frac{(e H) m^{2}}{4 \pi^{2}} \int_{0}^{\infty} d s \mathrm{e}^{-s m^{2}}\left(\operatorname{coth}(e H s)-\frac{1}{e H s}\right) .
$$

The terms that do not dependent on the field $H$ were taken away by renormalization. form

This result can be easily rewritten for a more general option with an axial condensate given in the

$$
b=\left(b_{0}, 0,0, b_{3}\right), \text { where both } \mathrm{b}_{0} \neq 0, \mathrm{~b}_{3} \neq 0 .
$$

Then, by introducing a vector $\beta^{\mu}=\frac{1}{2} \varepsilon^{\mu \nu \alpha \beta} b_{v} F_{\alpha \beta}$, with nonzero components $\beta^{0}=\varepsilon^{0312} b_{3} F_{12}=$ $b_{3} H, \quad \beta^{3}=\varepsilon^{3012} b_{0} F_{12}=-b_{0} H$, we deal with the same intermediate expressions as in the previous situations, and the unified result can then be represented in the invariant form

$$
\Delta \mathcal{L}^{b}=-\beta^{2} \frac{e^{2} m^{2}}{4 \pi^{2}} \int_{0}^{\infty} d s \mathrm{e}^{-s m^{2}}\left(\frac{\operatorname{coth}(e H s)}{e H}-\frac{1}{(e H)^{2} s}\right),
$$

here $\beta^{2}=\beta^{\mu} \beta_{\mu}$ and $H$ is an invariant quantity equal to the strength of a magnetic field in the system, where $\vec{H} \| O z$.

c) Consider the eigenvalue solution $\Delta \mu \neq 0, \vec{b}=0, b_{0}=0$

Assuming $\Delta \mu H$ to be small, consider the expansion up to $(\Delta \mu H)^{2}$ in (9) for $\Delta \mathcal{L}$. In what follows we shall use the notation $\Delta \mu H=h$. The linear in $h$ contribution for $n \neq 0$ is vanishing, since the expression for $\Delta \mathcal{L}$ is odd with respect to $\zeta= \pm 1$. However, contribution of the ground state $(n=0)$ is nonzero, since the sign is fixed: $\zeta=+1$ (for positively charged femion) or $\zeta=-1$ (for negatively charged femion).

Let us first consider the terms that are proportional to $h^{2}$.

After regularization and renormalization by addition of the appropriate counter terms, the final total expression can be found in the following form: ${ }^{3}$

$$
\Delta \mathcal{L}^{h q}=-\frac{h^{2} e H}{2(2 \pi)^{2}} \int_{0}^{\infty} d s \frac{\mathrm{e}^{-s m^{2}}}{s}\left(\operatorname{coth}(e H s)-\frac{1}{e H s}\right)+\frac{h^{2}}{2(2 \pi)^{2}}\left(\Lambda^{2}+m^{2} \log \frac{\Lambda^{2}}{m^{2}}\right) .
$$

Consider the contribution from the linear term

$$
\Delta \mathcal{L}^{h l}=h \frac{e H m}{(2 \pi)^{2}} \int_{\frac{1}{\Lambda^{2}}}^{\infty} \frac{d s}{s} \mathrm{e}^{-s m^{2}}=h \frac{e H m}{(2 \pi)^{2}} \log \frac{\Lambda^{2}}{m^{2}} .
$$

Recall that $\Delta \mu \simeq \kappa \mu_{0}$, then one may rewrite the counterterms for $\Delta \mathcal{L}^{h q}$ in the form

$$
\frac{h^{2}}{2(2 \pi)^{2}}\left(\Lambda^{2}+m^{2} \log \frac{\Lambda^{2}}{m^{2}}\right)=\left(\frac{\kappa}{4(2 \pi)}\right)^{2}(e H)^{2}\left(\log \frac{\Lambda^{2}}{m^{2}}+\frac{\Lambda^{2}}{m^{2}}\right) .
$$

At the same time, we have for the linear term

\footnotetext{
${ }^{3}$ This result was obtained earlier in [31], where only terms qudratic in $\Delta \mu$ were observed
} 


$$
\Delta \mathcal{L}^{h l}=h \frac{e H m}{(2 \pi)^{2}} \int_{\frac{1}{\Lambda^{2}}}^{\infty} \frac{d s}{s} \mathrm{e}^{-s m^{2}}=h \frac{e H m}{(2 \pi)^{2}} \log \frac{\Lambda^{2}}{m^{2}}=\frac{\kappa}{2(2 \pi)^{2}}(e H)^{2} \log \frac{\Lambda^{2}}{m^{2}} .
$$

Thus, the divergences in the effective Lagrangian $\Delta \mathcal{L}^{h}=\Delta \mathcal{L}^{h q}+\Delta \mathcal{L}^{h l}$ could be eliminated by making renormalization for the factor $\kappa$ in the anomalous magnetic moment expression, yielding the final result

$$
\Delta \mathcal{L}^{h}=-\frac{h^{2} e H}{2(2 \pi)^{2}} \int_{0}^{\infty} d s \frac{\mathrm{e}^{-s m^{2}}}{s}\left(\operatorname{coth}(e H s)-\frac{1}{e H s}\right) .
$$

d) Consider the eigenvalue solution $h \neq 0, \vec{b}=0, b_{0} \neq 0$

In this more general case we shall follow [15] and introduce, for convenience, a mixing angle $\Theta$, and we go over to the effective mass $\widetilde{m}$ and momentum $\widetilde{p}$ :

$$
\Theta=\arctan \frac{b_{0}}{h}, \quad\left(\begin{array}{c}
\widetilde{m} \\
\widetilde{p}
\end{array}\right)=\left(\begin{array}{cc}
\cos \Theta & \sin \Theta \\
-\sin \Theta & \cos \Theta
\end{array}\right)\left(\begin{array}{c}
m \\
p
\end{array}\right), \quad\left(\begin{array}{c}
m \\
p
\end{array}\right)=\left(\begin{array}{cc}
\cos \Theta & -\sin \Theta \\
\sin \Theta & \cos \Theta
\end{array}\right)\left(\begin{array}{c}
\widetilde{m} \\
\widetilde{p}
\end{array}\right) .
$$

The energy spectrum (8) can be written in these new effective variables as follows:

$$
E^{2}=\left(\sqrt{\widetilde{m}^{2}+2 e H n}+\widetilde{\mu} H \zeta\right)^{2}+\widetilde{p}^{2} .
$$

We shall name the quantity $\widetilde{\mu}$ as an effective anomalous magnetic moment. Taking the anomaly of the ground state into account, we consider the cases $n \neq 0$ and $n=0$ separately. In the last case, the spectrum of the ground state is rewritten in terms of the effective variables, as follows:

$$
E^{2}=(-\widetilde{m}+\widetilde{\mu} H)^{2}+\widetilde{p}^{2} .
$$

Expanding expression (9) in small parameter $\widetilde{\mu} H$ and calculating the integral over momentum, the contributions to the effective Lagrangian linear with respect to $\widetilde{\mu} H$ can be written as

$$
\Delta \mathcal{L}^{\tilde{\mu} H, l}=\frac{(e H)(\tilde{\mu} H) m}{(2 \pi)^{2}} \cos \Theta \int_{\frac{1}{\Lambda^{2}}}^{\infty} \frac{d s}{s} \mathrm{e}^{-s m^{2}} .
$$

This linear in $\widetilde{\mu} H$ contribution has the same divergence as in case c) Eq. (20).

After summing the terms of the $\Delta \mathcal{L}$ expansion quadratic in $\widetilde{\mu} H$ including quadratic contributions both from AMM $\Delta \mu$ and the parameter violating Lorentz invariance $b$, the final renormalized expression for the effective Lagrangian has the form

$$
\Delta \mathcal{L}^{q}=-(\widetilde{\mu} H)^{2} \frac{e H}{(2 \pi)^{2}} \int_{0}^{+\infty} d s \mathrm{e}^{-m^{2} s}\left(m^{2} \sin ^{2} \Theta+\frac{1}{2 s} \cos ^{2} \Theta\right)\left(\operatorname{coth}(e H s)-\frac{1}{e H s}\right),
$$

where the renormalization is performed similar to case $\mathbf{c})$.

Note that the final expression is correctly transformed into particular cases $\mathbf{c})(\mu \neq 0$ and $b=0)$ or a) $\left(b_{0} \neq 0\right.$ and $\left.\mu=0\right)$ :

$\underline{\Theta=0}$ leads to the variant $b=0$, and $\tilde{\mu} H=h$

$$
\Delta \mathcal{L}=\Delta \mathcal{L}^{h}=-\frac{1}{2} h^{2} \frac{e H}{(2 \pi)^{2}} \int_{0}^{+\infty} \frac{d s}{s} \mathrm{e}^{-m^{2} s}\left(\operatorname{coth}(e H s)-\frac{1}{e H s}\right) .
$$

$\Theta=\frac{\pi}{2}$ leads to the variant $\mu=0$, and $\widetilde{\mu} H=b_{0}$

$$
\Delta \mathcal{L}=\Delta \mathcal{L}^{b}=-b_{0}^{2} \frac{e H}{(2 \pi)^{2}} m^{2} \int_{0}^{+\infty} d s \mathrm{e}^{-m^{2} s}\left(\operatorname{coth}(e H s)-\frac{1}{e H s}\right) .
$$




\section{Conclusions}

In this report, we calculated the first nonvanishing contributions to the effective Lagrangian, taking into account the interaction of a fermion with an axial vector and an anomalous magnetic moment in an external magnetic field. These parameters may be considered as some sort of condensate values that follow from higher dimensional fundamental interactions. The results of this short report will be extended and generalized in our forthcoming publications to other possible effects of the nontrivial structure of the QFT vacuum and its manifestations, such as the chiral magnetic effects, and others.

\section{References}

[1] Schwinger J. // Proc. Nat. Acad. Sci. USA, 40, 132 (1954).

[2] Ternov I.M., Bagrov V.R., Dorofeyev O.F., Khalilov V.R., Rodionov V.N. // J. Phys. A: Math. Gen. 1978 11. P. 739

[3] Roberts B. M., Stadnik Y. V., Dzuba V. A., Flambaum V., Leefer N., and Budker D. // arXiv: $1409.2564 \mathrm{v} 2$

[4] Pauli W. // Rev. Mod. Phys, 1941, 13, P. 203

[5] Ternov I.M., Zhukovsky V.Ch., Borisov A.V. // Quantum processes in a strong external field (in Russian): (Moscow University 1989)

[6] Shapiro I. L. // Phys. Rept. 2002 357, P. 113

[7] Ahmadi F., Jalalzadeh S., Sepangi H. R. // Class. Quantum Grav. 2006 23. P. 4069

[8] Bertolami O., Carvalho C. // Phys. Rev. D 2006 74. P. 084020

[9] Kostelecky V.A., Samuel S. // Phys. Rev. D. 1989 39. P. 683

[10] Colladay D., Kostelecky V.A. // Phys. Rev. D. 1998 58. P. 116002

[11] Jackiw R., Kostelecky V.A. // Phys. Rev. Lett. 1999 82. P. 3572

[12] Zhukovsky V.Ch., Lobanov A.E., Murchikova E.M. // Phys. Rev. D. 2006 73. P. 065016

[13] Lobanov A. E., Murchikova E. M. // Univ. Phys. (2008) 63: 91

[14] Ebert D., Zhukovsky V. Ch., and Razumovsky A. S. // Phys. Rev. D 2004 70, P. 025003

[15] Frolov I.E., Zhukovsky V.Ch. // J. Phys. A. 2007 40. P. 10625

[16] Roberts M., Stadnik Y. V. // Phys. Rev. D. 2014 90. P. 096005

[17] Fukushima K., Kharzeev D.E., Warringa H.J. // Phys. Rev. D 2008 78. P. 074033

[18] Chernodub M. N. et.al. // hep-th 1311.0878v2

[19] Chernodub M. N., Zubkov M. // hep-th 1508.03114v1

[20] Braguta V. et.al. // EPJ Web of Conferences 95, 03002 (2015)

[21] Bezerra de Mello E. R. // JHEP 0406, 016 (2004).

[22] Kadyshevsky V. G. and Rodionov V. N. // Theor. and Math. Phys. 20031363 P. 1346

[23] Gitman D. M. and Saa A. V. // Class. Quant. Grav. 10, 1447 (1993).

[24] Kharlanov O. G., and Zhukovsky V. Ch // J. of Math.Phys. 200748.9 P. 092302

[25] Frolov I. E., Zhukovsky V. Ch., Klimenko K. G. // Phys. Rev. D 2010 82, P. 076002

[26] Sitenko Y.A., Rulik K.Y. // Eur. Phys. J. 2003 C28. P. 405

[27] Bubnov A. F., Zhukovsky V. CH. // Moscow Univ. Phys. (2010) 65: 105

[28] Ternov I.M., Bagrov V.G., Zhukovsky V.Ch. // Moscow Univ. Bull., 1966. 21. P. 21.

[29] Rodionov V. N., J. of Exp. and Theor. Phys. 2004. 98. P. 395

[30] Fock V. A. // Sow. Phys. 1937 12. P. 404.

[31] O’Connell R. F. // Phys. Rev. 1968 176. n.5. P. 1433. 